\title{
A re-examination of throats
}

\author{
J.-W. Kim, ${ }^{1}$ D. Kim, ${ }^{2}$ and W. B. Lindquist ${ }^{1}$ \\ Received 12 June 2013; revised 8 October 2013; accepted 28 October 2013; published 20 November 2013; corrected 30 January 2014.
}

[1] We critically re-examine the concept of a throat in a porous medium as a geometric quantity defined independently of an entry meniscus in a drainage process. To maintain the standard notion of a throat as a locally minimum-area cross section in the pore network, we demonstrate with examples that throats must intersect each other. Using flow simulation, we show that these intersecting throats correspond to capillary pressure controlled entry points during drainage. We have designed a throat-finding algorithm that explicitly locates intersecting throats, using a planar approximation for robustness and speed. The capability of the new algorithm was compared against an existing algorithm in the construction of pore-throat networks from X-ray computed tomography images of consolidated sandstones ( $7.5-22 \%$ porosity) and of an unconsolidated sand pack (32.5\% porosity). We show that the probability of throat intersection increases significantly with porosity above $20 \%$; in the sand pack, over $1 / 4$ of all throats intersect with another.

Citation: Kim, J.-W., D. Kim, and W. B. Lindquist (2013), A re-examination of throats, Water Resour. Res., 49, 7615-7626, doi:10.1002/2013WR014254.

\section{Introduction}

[2] Throats play a fundamental role in the two-phase flow drainage process: in the vadose zone (air-water) and in the saturated zone (where the two-phase drainage process is, almost universally, organic-water with the organic phase being either liquid or gas) [Bear, 1972; Dullien, 1992]. By extension, throats are equally important in threephase flow. Throats also play a strong role in determining single-phase conductivity [Sholokova et al., 2009]. Throats locally control the movement of the terminal menisci that define the invading fluid front and throat shape plays a role in the formation of the arc menisci that define wetting films [Frette and Helland, 2010; Glantz and Hilpert, 2007; Lindquist, 2006; Mayer and Stowe, 1965; Morrow, 1970; Princen, 1969a]. Pore-network models [Blunt, 2001] are one of the main numerical tools used to model flow at the pore-scale. These models require increasingly accurate characterization of pore bodies and pore-pore connectivity in order to compute increasingly complex multiphase drainage (and imbibition) flows. Throat characterization plays an important role in pore-pore connectivity.

[3] Under quasistatic flow, which we assume for this article, the drainage process (a nonwetting fluid $f_{n}$ displacing a wetting fluid $f_{w}$ ) can be viewed as a mapping between the capillary pressure (the pressure change across the fluid-

\footnotetext{
${ }^{1}$ Department of Applied Mathematics and Statistics, Stony Brook University, Stony Brook, New York, USA.

${ }^{2}$ King Abdullah University of Science and Technology, Thuwal, Saudi Arabia.

In the originally published version of this article the rows in Figure $6 \mathrm{~b}$ were inverted. Figure 6 and its caption have been corrected on 30 January 2014.

Corresponding author: W. B. Lindquist, Department of Applied Mathematics and Statistics, Stony Brook University, Stony Brook, NY 117943600, USA. (lindquis@ams.sunysb.edu)

fluid surface) and the locations of the advancing fluid-fluid menisci (both terminal- and arc-menisci) within the pore network. At any given capillary pressure, $P_{C}$, the terminal menisci are at locations where the local geometry of the pore network supports a stable fluid-fluid meniscus of radius $r$ given by the Young-Laplace equation

$$
P_{C}=2 \sigma \cos \theta / r
$$

where $\sigma$ is the surface tension between the two fluids and $\theta$ is the wetting angle determined by the two fluids and the nature of the grain surface. (For simplicity, we have written the Young-Laplace equation as for cylindrical geometries rather than using the more general form with two principle radii of curvature. This simplification does not affect the point of the discussion - the existence of entry menisci.) Usually an $O(\epsilon)$ increase in capillary pressure results in an $O(\epsilon)$ displacement in a terminal meniscus and an $O(\epsilon)$ volumetric displacement of $f_{w}$ by $f_{n}$. However, when a terminal meniscus reaches a "throat position," an $O(\epsilon)$ increase in capillary pressure eventually results in an $O(1)$ displacement of the terminal meniscus until the meniscus again finds itself in a stable location. (Displacements may be accompanied by the formation of wetting films and an $O(1)$ displacement may be accompanied by splitting of the terminal meniscus or even merger with another terminal meniscus.) An $O(1)$ displacement is known as a Haines jump [Morrow, 1970] and results in a measureable $O(1)$ volumetric displacement of $f_{w}$ by $f_{n}$. We refer to the stable terminal meniscus immediately prior to a Haines jump as an "entry" meniscus; the capillary pressure causing the jump is referred to as the entry pressure $P_{C}^{e}$, which is related to the radius of curvature $r_{e}$ of the entry meniscus by equation (1). Since the Haines jump occurs at constant value of $P_{C}^{e}$, the jump must terminate with the terminal meniscus at the first downstream location that supports a stable meniscus having radius of curvature $r_{e}$. 
[4] The capillary pressure must monotonically increase to continue to drive the displacement process. As is well known from mercury porosimetry measurements [e.g., Dullien, 1992], micromodel experiments [e.g., Lenormand et al., 1983], theory [e.g., Mayer and Stowe, 1965], and computational simulations [e.g., Joekar-Niasar et al., 2009; Koplik and Lasseter, 1985; van der Marck et al., 1997], successive Haines jumps can only occur at positions corresponding to a decreasing sequence of values of $r_{e}$. Thus, the drainage process is characterized by a sequence of pre-Haines-jump positions in the pore network referred to as "throats" and the concept of a throat derives its physical significance from the drainage process. We make the following observation:

[5] (D0) The position of an "entry" meniscus under quasistatic drainage provides an experimentally measureable determination of a throat location in a pore network.

[6] Observation D0 does not precisely define the throat; the natural definition derived from $\mathrm{D} 0$ is:

[7] $\left(\mathrm{D}^{\prime}\right)$ A throat is identically the entry meniscus surface.

[8] In everyday practice in the field of porous media, however, we have come to think of a throat as an intrinsic geometrical property of the pore network structure. We make statements such as "drainage is governed by the smallest throats and imbibition by the largest pores," implying that a pore network structure intrinsically contains throats that "govern"-rather than "are defined by"-drainage processes. This practice prompts us to critically re-examine the throat concept. In the remainder of the discussion we shall reserve the word "throat" for a drainage-independent, geometric quantity and consider the following two questions. (1) How completely can a throat be defined in a manner independent of a particular drainage process? (2) What is the precise characterization of a throat?

[9] Regarding the first question, throats can only be defined independently of drainage if the throat location does not change from one drainage process to the next. This is problematic for two reasons. As shown in Figure 1, the location (and shape) of the entry meniscus changes somewhat with wetting angle. This variation is arguably small and it is reasonable to require that the location of a throat be within the range of entry meniscus positions

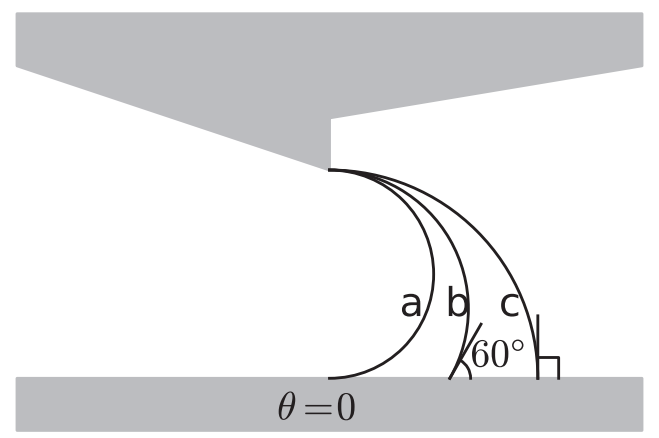

Figure 1. Meniscus position prior to Haines filling of the downstream (right hand) pore during drainage depends on wetting angle, as illustrated here for wetting angles $0^{\circ}, 60^{\circ}$, and $90^{\circ}$. Void space is white, grain is gray. exhibited under variation of wetting angle. As drainage can occur in either direction down a channel, it is reasonable to require that

[10] (T1) a throat approximates an average entry meniscus location, where the average is over wetting angles and possible flow directions.

[11] As mentioned, there is a second problem. In the channel cross sections sketched in Figure 2 (cylindrical symmetry assumed for simplicity), the location indicated by "a" will be the only entry meniscus location for any drainage process through the channel moving from left to right, while the locations indicated by " $a$ " and " $b$ " will be the two entry meniscus locations for any drainage process moving through the channel from right to left. If throats are to be defined independently of a drainage process, then both locations " $a$ " and "b" are throats and we must accept that

[12] (T2) not all throats will contribute in every drainage process.

[13] T1 and T2 decouple the definition of a throat from the drainage process. These observations reinforce the following definition:

[14] (D1) a throat is a position of local minimum crosssectional area in the void network. D1 concurs with the general understanding of a throat [Bear, 1972; Dullien, 1992; Gao et al., 2012; Kovscek and Radke, 1996; Raoof and Hassanizadeh, 2012; Shaeri et al., 2013].

[15] The advent of X-ray computed microtomography (XCMT) at energies and luminosities sufficient to produce 3-D images of geologic samples has spurred the development of a number of computational throat-finding algorithms [e.g., Glantz and Hilpert, 2007, 2008; Lindquist and Venkatarangan, 1999; Shin et al., 2005; Silin and Patzek, 2006], all of which define throats based upon definition D1. This generally accepted definition requires some ad hoc decision making to implement. By straightforward application of D1, each of the locations " $a$ " and " $b$ " in Figure $2 b$ acts as a throat in a drainage process invading from the right. However, it can be argued that location " $b$ " is not a "significant" throat, being merely a minor perturbation in the local throat cross-sectional area. (Note, however, that, in a careful porosimetry experiment, an $O(\epsilon)$ pressure increase above the entry pressure associated with location "b" will result in a sizable right to left meniscus motion.)

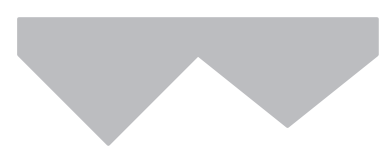

a

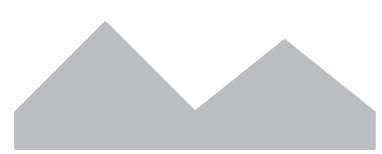

(a)

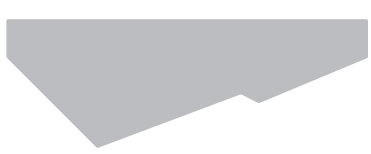

a

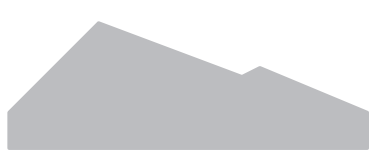

(b)
Figure 2. (a) For drainage from left to right, only the constriction "a" acts as a throat. For drainage from right to left, both constrictions "a" and "b" act as throats. (b) Here constriction "b" is less "significant." Void space is white, grain is gray. 
Given an accurate depiction of the roughly surfaced, irregular geometry in real geologic porous media, it is easy to see that "minor" local minima in the cross section occur constantly. There is no convention regarding what is "minor"; in practice, throat-finding algorithms make individual decisions on which "minor" local minima they will ignore. (e.g., the maximal ball method of Silin and Patzek [2006] uses a well-defined overlapping-ball-centers criterion.) Glantz and Hilpert [2008] suggest that persistent homology [Edelsbrunner and Harer, 2008] may provide an answer to this question. This is an area to be investigated.

[16] We now address the second question, what quantities should be used to characterize a throat? Is it only a location? - in which case it would be properly described by a perimeter, that is by a topological ring inscribed on the grain surface "circling" the channel at the appropriate location. Mayer-Stowe-Princen (MSP) theory, which analytically specifies the quasistatic flow condition on $r_{e}$ for piston-like entry of $f_{n}$ into a channel of constant crosssectional area occupied by $f_{w}$, contains both perimeter and area terms related to the invading $f_{n}$ [Mayer and Stowe, 1965; Princen, 1969a, 1969b]. Approximations to this theory, such as Hwang's [1977] hydraulic radius, strongly suggest that the ratio $A / P$ of the throat area, $A$, and perimeter, $P$, captures most of the variance in predicting $r_{e}$ for realistic channels [Lindquist, 2006]. (Alternately expressing Hwang's radius as $r_{e}=G P$ suggests that the perimeter and the dimensionless shape factor $G$ are the two primary throat characterizations. To first order, $G=A / P^{2}$.) Note that in MSP theory, the area of the (curved) entry meniscus surface does not enter into consideration. However, MSP theory deals with channels of constant cross section, which reduces to a planar 2-D problem. In extrapolating to 3-D, we propose the above observations argue that:

[17] (T3) the appropriate characterizations of a throat are the area of the local minimal-area cross section and its perimeter (which defines its location).

[18] Under T3 note that if the throat surface is simply connected, it will have a single perimeter (a topological ring inscribed on the grain surface circling the channel). However, if the throat surface is not simply connected, its perimeter consists of a union of $n$ disjoint "rings," $n-1$ of these rings encircling "grain intrusions" piercing the throat surface. A throat-finding algorithm must be capable of handling both connectivity possibilities.

[19] Having identified the appropriate throat quantities, one is still left with the problem of computing them. As example, the throat-finding algorithm in Lindquist and Venkatarangan [1999] locates a throat by finding a minimum length perimeter (having unit winding number) for each channel (or appropriate subsection of each channel). Given the perimeter, its minimum spanning area is approximated by a triangulation centered on a point assumed to be the center of the throat surface. In Shin et al. [2005], the cross-sectional area is minimized, after which the perimeter of the minimum area is computed. In Silin and Patzek [2006], the center, circumference, and circumference area of a locally minimumradius maximally inscribed ball defines a throat. Under definition D1, the main difficulty in computing minimal-area cross sections is that they are, in general, nonplanar. This is demonstrated by an artificial example (Figure 3) which shows two partially overlapping cylindrical pores drilled at

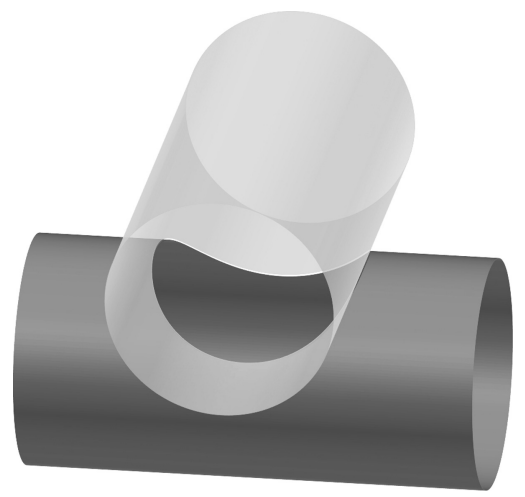

Figure 3. A nonplanar "saddle shaped" throat formed by the intersection of two cylindrical pores. The interior of the cylinders represents void space; the exterior is grain.

right angles to each other. The computational work needed to accurately compute the required minimal area "saddleshaped" surface would be prohibitive for software that must compute tens of thousands of throat locations in typical 3-D XCMT images of geologic porous media. Consequently, the minimum-area cross sectional required in definition D1 must typically be approximated.

[20] In general, we have found that existing throatfinding algorithms work acceptably (i) for geologic media with porosities $20 \%$ or less and (ii) in special cases of highporosity media if pores are very regular in shape [Shin et al., 2005]. However, for geologic media exceeding 20\% porosity, especially unconsolidated material, we find that existing algorithms do not explicitly account for the fact that:

[21] (T4) throats may intersect each other.

[22] Referring to D0, which provides the experimental determination of throat locations in terms of entry menisci, we note that terminal menisci do frequently touch one another (intersect), either as a result of a "merge" or a "split" process. It is not hard to construct an example such as Figure 4 which sketches two drainage entry menisci, $M_{1}$ and $M_{2}$, intersecting at their respective channel entrances to pore $P$. For physical stability, the two menisci are also in contact with the grain surface along their respective lineof-contact. The entrance of meniscus $M_{1}$ into $P$ will destabilize $M_{2}$ and a merged terminal meniscus $M$ will sweep through pore $P$. Since definition D1 removes the precise

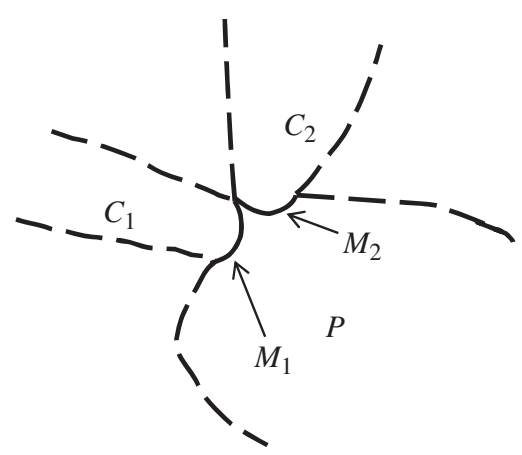

Figure 4. Sketch of the intersection of two entry menisci, $M_{1}$ and $M_{2}$ prior to $f_{n}$ filling pore $P$. 
correlation (characteristics T1 and T2) between a drainage process and a throat, throats now become geometrical (rather than physical) objects and there is no physical requirement that throat surfaces intersect each other only along a curve coincident with a grain boundary. We illustrate throat intersection with an artificial example (Figure 5). We claim that the pores labeled 1 and 2 are separated by a throat, so are pores 2 and 3, 3 and 4, and 4 and 1, and that these throats border one another (intersect).

[23] The intersection of throat surfaces seems to be confirmed by the "tight dual model" geometric throat construction method of Glantz and Hilpert [2008] which produces throats that are unions of two dimensional Delaunay cells. The computation in Figure $18 \mathrm{c}$ of that paper shows two throats which share a common Delaunay cell. Referring to our condition T5 later on in this paper, we would reinterpret this configuration as three throat surfaces meeting along a common cell boundary.

[24] The goal of the discussion in this section has been to derive geometrical requirements for throats from the physics of capillary displacement. In section 2, we discuss a simulated drainage process whose Haines jumps clearly demonstrate the existence of intersecting throats. The computations in section 2 demonstrate that, under drainage, when two (or more) throats intersect, each acts independently as a capillary barrier in the direction perpendicular to that throat. In section 3 , we develop a throat-finding algorithm that allows for the existence of intersecting throats and handles throat surfaces that may - or may not-be simply connected. In section 4, we discuss the application of this algorithm to Figure 5, demonstrating by construction

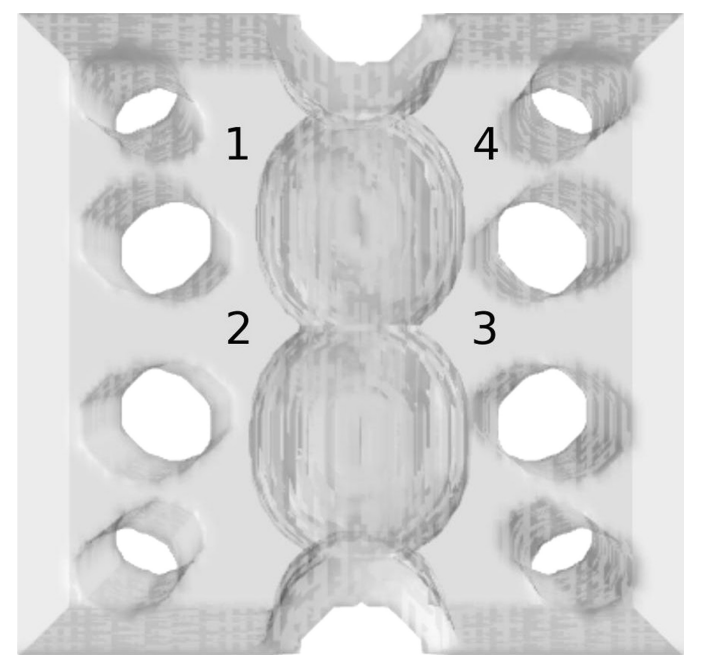

Figure 5. An artificial porous medium consisting of a back and front plate joined by eight solid cylinders, four on the left, four on the right. For representation purposes, the insides of the solid cylinders are not shown-only the cylinder surface. A "column" of two solid spheres "welded" to each other are also "welded" to half-sphere indentations on the bottom and top plates. These two spheres do not contact the front and back plates. There is void space between the plates, cylinders, and spheres. The sample can be sealed on either pair of opposing sides to examine flow through the medium. The numbered regions indicate the positions of four of the interior pores. that these throats do intersect each other. In section 5, we discuss the results of the application of our algorithm to XCMT images of Fontainebleau sandstones as well as an unconsolidated soil sample, comparing the performance of this new algorithm against the algorithm described in Prodanovic et al. [2006]. This latter algorithm, hereafter referred to as PLS, combines the techniques described in Lindquist and Venkatarangan [1999] and Shin et al. [2005].

\section{Drainage Simulation}

[25] LSMPQS [Prodanovic and Bryant, 2006] is a software package that simulates capillary controlled, quasistatic, two-phase immiscible fluid displacement in 3-D using the level set method. We utilized LSMPQS to simulate drainage at a contact angle of $0^{\circ}\left(f_{w}\right.$ is perfectly wetting). To begin, we simplified the porous medium of Figure 5 further, simulating a representation of the "front half" of Figure 5 by a rectangular network whose dimensions are easier to control. Front and side views of the simplified network are presented in Figure 6. The six rectangular pores were connected by horizontal and vertical channels. Each channel had rectangular shape, and constant cross section with independently assigned heights (visible in the side view in Figure 6b). Note that the channels interconnect. As each channel had constant cross-sectional shape there was no unique location for the throat in a channel; each channel acted as a throat. Consequently, the throats interconnect. In referring to Figure 6, we will use "channel" and "throat" interchangeably.

[26] Figures 7a-7d show the LSMPQS drainage simulation with the fluid $f_{n}$ (darker fluid) entering the network from a reservoir connected to pore P2. Fluid exited the network into a reservoir connected to pores P4, P5, and P6 on the right. Initially, the capillary barriers due to the throats $\mathrm{C} 12, \mathrm{C} 25$, and $\mathrm{C} 23$ retained $f_{n}$ in P2 (Figure 7a). The first Haines jump occurred into P1 (Figure 7b). Throat sizes were such that the jumps into P5 and P6 occurred within

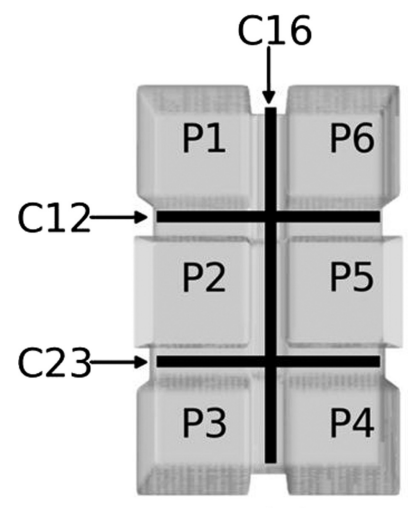

(a)

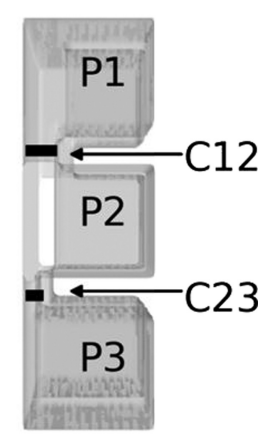

(b)
Figure 6. (a) Front and (b) side views of a rectangular six-pore network joined by vertical and horizontal channels. The pores are labeled P1 through P6. The channel connecting pores $i$ and $j$ is labeled $C i j$. The connecting channels $\mathrm{C} 12, \mathrm{C} 23$, and $\mathrm{C} 16$ are indicated. Throats are indicated by solid lines. C12 has a larger throat than $\mathrm{C} 23$. Throats in channels C12, C16, C56, and C25 intersect. 


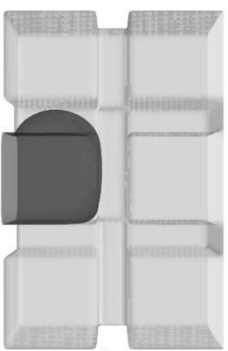

(a)

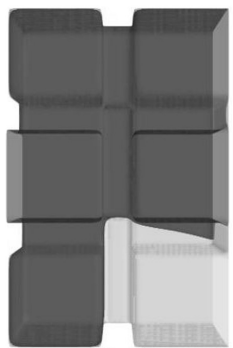

(c)

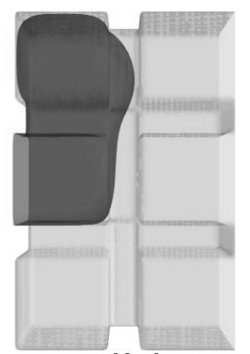

(b)

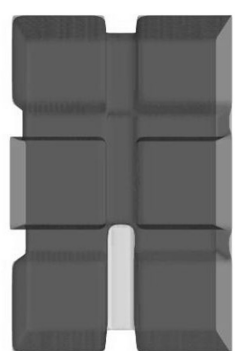

(d)

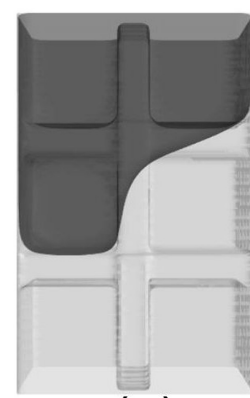

(e)
Figure 7. Snapshots of an LSMPQS drainage simulation through the pore network of Figure 6. $(\mathrm{a}-\mathrm{d})$ Pore P2 was connected to an inlet reservoir holding $f_{n}$ (dark fluid); pores P4, P5, and P6 were connected to an outlet reservoir. (e) A single snapshot of a simulation in which pores P1 and P6 were connected to the inlet reservoir and pores P3 and P4 were connected to the outlet reservoir.

the $\Delta P_{C}$ chosen as the capillary pressure change driving each step of the drainage simulation. Throat $\mathrm{C} 23$ was the site of the next Haines jump (Figure 7c), followed by $\mathrm{C} 45$ (Figure 7d). In Figure 7d, C34 had yet to be filled with $f_{n}$. Figure 7e shows a snapshot of a vertical drainage simulation through the same network. Here the order of throat breakthrough was C16, C12 (Figure 7e), C25/C56, C23, and $\mathrm{C} 45$. In particular, note in Figures $7 \mathrm{a}, 7 \mathrm{c}$, and $7 \mathrm{e}$ how throats, intersected at right angles to each other, correspond to locations where the drainage interface is held in check.

[27] Accepting T4 leads to the understanding that complex geometric scenarios can occur, three of which are sketched in Figure 8, showing two (Figure 8a), three (Figure $8 \mathrm{~b}$ ), and four (Figure 8c) intersecting throats. Examination of Figure 8c opens another question. Does this 2-D sketch represent four throat surfaces (as labeled) that meet along a common line (in 3-D), or are these two throat surfaces that pass through each other. Based upon the example of Figure 6 , we propose a fifth throat condition:

[28] (T5) a single throat must uniquely separate two distinct pores.

[29] Condition T5 argues for the throat labeling shown in Figure 8 .

\section{An Algorithm for Intersecting Throats}

[30] To our knowledge, none of the existing throatfinding algorithms makes any systematic effort to account for the possibility of intersecting throats. To address this, we have developed a new throat-finding algorithm for the 3DMA-Rock package which explicitly allows for this necessity.

[31] 3DMA-Rock uses a medial axis network [Lee et al., 1994] computed in the void space of a segmented [Oh and Lindquist, 1999] 3-D XCMT image as a searchable network for the purpose of throat finding. Prior to throat finding, the medial axis is processed to eliminate from consideration branches of the network upon which no throats are expected to be found, either because they are "dead end" paths, or they exhibit a measure that indicates they lie entirely within a single pore body [Lindquist and Venkatarangan, 1999]. After this processing, each remaining branch of the medial axis is a likely candidate to contain one or more throats.

[32] Our new algorithm makes a concession for speed and robustness by approximating throats by planar surfaces. As discussed in section 1, throat surfaces are generally expected to have curvature. Consequently, we compared the results of the new algorithm against computations performed by the existing PLS algorithm which does allow for curved throats. The heart of the new algorithm is the ability to rapidly construct a "plane" of 6-connected voxels at an arbitrary $\theta, \varphi$ (spherical angle coordinates) orientation passing through a specific medial axis point. We refer to this set of voxels as a throat barrier. (3DMA-Rock treats the void space in a digitized image as 26-connected. To separate pores, the throat barrier must be a 6-connected assemblage of voxels.) The barrier contains within it the

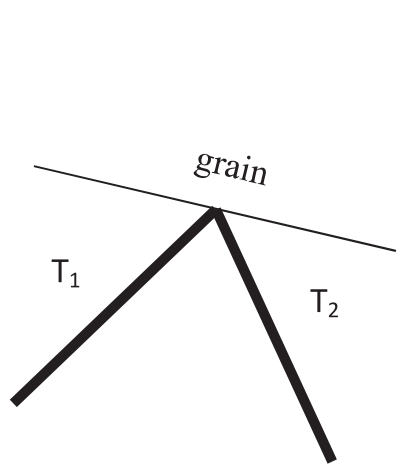

(a)

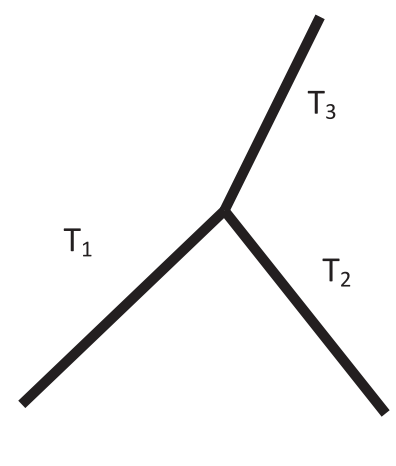

(b)

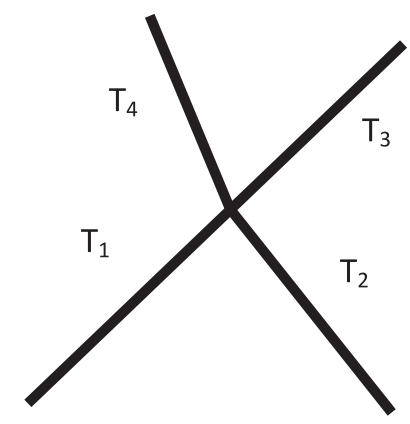

(c)

Figure 8. Possible scenarios for throat intersection include (a) two, (b) three, and (c) four throat intersections. 
analytic, 2-D planar throat surface whose perimeter and area will be used to characterize the throat.

[33] Two general considerations were built into the algorithm design. (1) Since, in drainage, smaller throats control the flow prior to larger ones the algorithm preferentially finds smaller area throats first. (2) However, if the algorithm needs to choose between two throats of similar area (we employ a $<5 \%$ area difference as a criterion for similar) in a channel, a nonintersecting throat is chosen in preference to one that intersects others.

[34] The core of the planar dilation algorithm comprises the construction, starting from any given "base" voxel on the medial axis, of a plane cross-section surface-a throat-and a 6-connected "barrier" of voxels, each such voxel intersecting the throat surface. Let $v$ denote the current "base" voxel. The center of $v$ is referred to as the base point. A least-squares fit using the base point and the center points of the six neighboring medial axis voxels to $v$ is used to establish the local tangential direction, $\hat{t}$, to the medial axis. The tangential direction acts as the zenith-axis in a spherical coordinate system centered at the base point. A polar angle $\theta$ relative to the zenith-axis, and an azimuthal angle $\varphi$ in the plane perpendicular to the zenith-axis (for fixed $\theta, \varphi$ is measured relative to the $x$ axis of the digitized medium) determine a normal vector, $\hat{n}_{\theta, \varphi}$. This normal vector is the normal to the plane $P_{v, t, \theta, \varphi}$ around which the throat barrier is constructed (Figure 9 ).

[35] Computation of the throat barrier proceeds as follows. Starting at $v$, we run a 26-connected dilation process through successive neighboring void voxels retaining only those that intersect the plane $P_{v, t, \theta, \varphi}$. Those so retained are the desired barrier voxels. The precise test for whether the plane passes through a void voxel $w$ involves the computation of eight inner products, $w_{i}, \quad i=1, \ldots, 8$, where $w_{i}$ is the inner product between the normal vector $\hat{n}_{\theta, \varphi}$ and the vector from the base point to corner $i$ of voxel $w$. If $w_{i}>0$, then corner $i$ lies above the plane; if $w_{i}<0$, corner $i$ lies below the plane; if $w_{i}=0$, corner $i$ lies on the plane. If

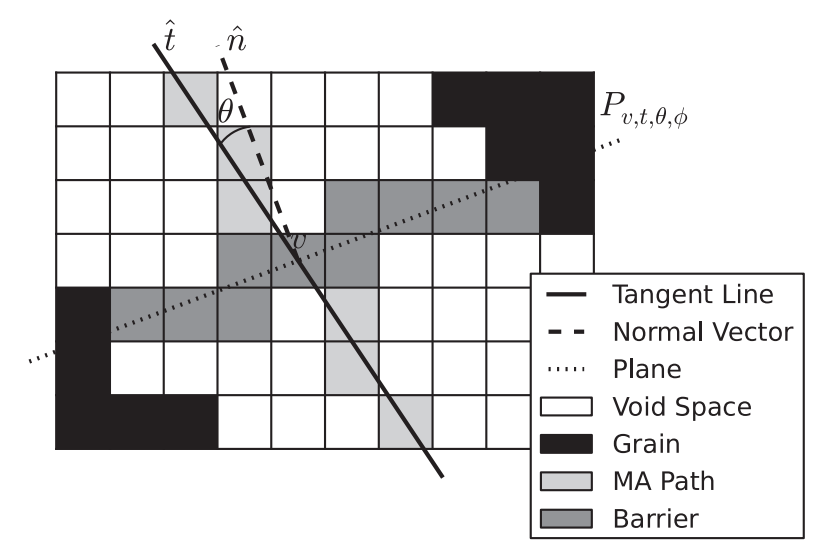

Figure 9. 2-D $(\varphi=0)$ illustration of the procedure used to find throat barriers. Voxel $v$ is the current medial axis voxel at which the computation is performed. $\hat{t}$ is the local tangent line to the medial axis at $v$; the vector $\hat{n}$, oriented at spherical angles $\theta, \varphi=0$ to $\hat{t}$, is the normal to the plane $P_{v, t, \theta, \varphi}$. The throat barrier voxels, all of whom are intersected by $P_{v, t, \theta, \varphi}$ must be 6-connected to separate the channel into two 26-connected void phases (pores). $w_{i}>0, \quad i=1, \ldots, 8$ or $w_{i}<0, \quad i=1, \ldots, 8$, then $P_{v, t, \theta, \varphi}$ does not pass through $w$, and $w$ is not a barrier voxel. If $w_{i}>0$ and $w_{j}<0$ for $j \neq i$, then $P_{v, t, \theta, \varphi}$ passes through $w$, and $w$ is taken to be a barrier voxel. Two cases remain: (a) $w_{i} \geq 0, \quad i=1, \ldots, 8$ or (b) $w_{i} \leq 0, \quad i=1, \ldots, 8$ with $w_{j}=0$ for at least one corner $j$. In case (a), we decide that $w$ is a barrier voxel; in case (b), we decide $w$ is not a barrier voxel. This choice guarantees that the barrier remains minimally 6-connected. (Allowing both case (a) and (b) to define a barrier voxel would produce barrier voxels whose removal would leave the barrier still 6-connected. Rejecting both case (a) and (b) as barrier voxels would generate barriers that were not 6-connected.) The dilation process, beginning from voxel $v$, terminates when all successively neighboring void voxels have been eliminated as possible barrier candidates.

[36] It is straightforward geometry to compute the area of the section of $P_{v, t, \theta, \varphi}$ that intersects each barrier voxel. The cross-section area of the throat is defined to be the sum of these sectional areas. It is similarly straightforward to compute the perimeter of the constructed throat. Consider the face between a barrier and a grain voxel that are 6connected. The intersection of this face and the plane $P_{v, t, \theta, \varphi}$ is a line segment. The union of all such line segments for a single throat barrier forms a "face polygon" that is a candidate for the perimeter of the throat. However, this polygon will have a decided "staircase" shape as it follows voxel faces (solid lines, Figure 10a). We therefore construct a second polygon by linearly connecting the midpoints of sequential line segments of the first polygon (dashed lines, Figure 10a). This second polygon is defined as the throat perimeter, and the throat area is recalculated based on this perimeter.

[37] Having explained how to compute a single (candidate) throat surface and corresponding (candidate) throat barrier for an arbitrary medial axis voxel, $v$, we describe how we compute a minimal area throat, and corresponding barrier, for a medial axis path, $p$. Again consider voxel $v$ on the path. Recall that the local tangent direction $\hat{t}$ at $v$ acts as the zenith-axis of a spherical coordinate system. Consider the sequence of normal vectors $\hat{n}_{i j}$ defined by the polar and azimuth angles $\theta_{i}, \varphi_{j}, \quad i=1, \ldots, m ; j=1, \ldots, n$. Each $\hat{n}_{i j}$ defines a plane $P_{v, t, \theta_{i}, \varphi_{j}}$ upon which to build a throat and its barrier through $v$. By varying normal vectors, we define a discrete optimization stencil over which we can search

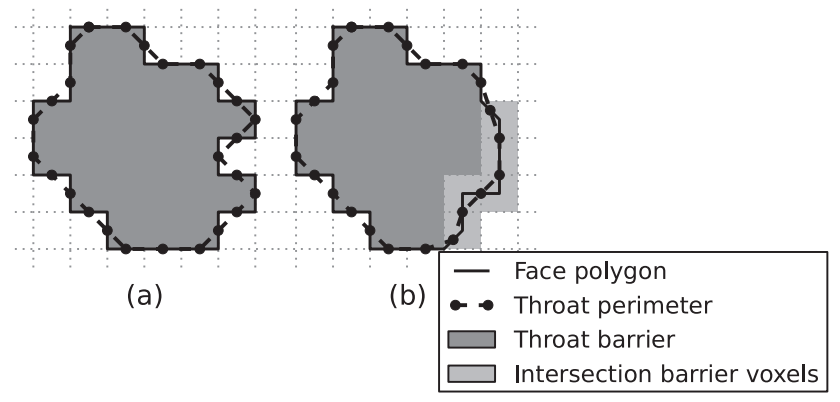

Figure 10. Illustration of perimeter construction for (a) a throat barrier that contacts grain voxels around its entire perimeter; (b) a throat barrier intersecting another throat barrier along part of its perimeter. The view is along the normal direction of the throat plane. 
for a minimal area throat/barrier candidate at $v$. (We used $m=9, n=8$ in our computations, with $5^{\circ} \leq \theta_{i} \leq 45^{\circ}$ in increments of $5^{\circ}$ and $0^{\circ} \leq \varphi_{j} \leq 315^{\circ}$ in increments of $45^{\circ}$. Together with the choice $\theta=\varphi=0$, this amounted to the construction of 73 throats/barriers at each voxel $v$ on each medial axis path. This required that the computation for each individual barrier be fast.) For voxel $v$, let $A_{v}=\min _{k=1, \ldots, 73} A_{k}$, where $A_{k}$ is the throat area computed for the $k$ th throat/barrier in the optimization stencil. Let $\theta_{v}, \varphi_{v}$ denote the normal direction corresponding to the throat having area $A_{v}$. For each voxel $v$ on a path, the information $v, \theta_{v}, \varphi_{v}, A_{v}, B_{v}$ is stored in a "cross-section" list. (Here, $B_{v}$ represents the information on the barrier voxels computed for this throat surface.) Thus, if the medial axis path $p$ is comprised of $n_{p}$ voxels, its cross-section list has length $n_{p}$.

[38] We enforce two conditions during throat finding.

[39] Condition 1: For the throat surface computed for any plane $P_{v, t, \theta_{i}, \varphi_{i}}$, we compute an effective throat radius $r(A)=\sqrt{A / \pi}$, where $A$ is the area of the throat surface. If $r(A)>\min \left(l_{s v}, l_{v e}\right)$, where $l_{s v}$ is the distance from the base voxel to the start of the medial axis path and $l_{v e}$ is the corresponding distance to the end of the medial axis path, then we ignore this as a throat possibility (i.e., no throat surface is computed for plane $\left.P_{v, t, \theta_{i}, \varphi_{j}}\right)$. Condition 1 is always applied during the planar dilation process described above. Condition 1 has two primary purposes. It neglects "minor" constrictions of the sort noted in Figure $2 b$ and it also prevents throat construction for a medial axis path that lies entirely within a single pore. As a result of enforcing condition 1, throat construction may "fail" on one or more (including all!) voxels of a path.

[40] Condition 2: This condition deals with throats that, constructed for one medial axis path, intersect a second medial axis path. Condition 2 is only applied to "declared" path throats (whose identification is described in the following paragraphs). Consider medial axis path $i$ having throat $t_{i}$ of area $A_{i}$ and effective radius $r\left(A_{i}\right)$. Assume $t_{i}$ intersects medial axis path $j(j \neq i)$ at voxel $v_{j}$. When the planar dilation algorithm runs on path $j$, those voxels of path $j$ lying within a distance $r\left(A_{i}\right)$ of $v_{j}$ are excluded from consideration. $r\left(A_{i}\right)$ is therefore an effective exclusion volume. If path $j$ is intersected by declared throats from several other paths, it is possible that the entire path $j$ lies in excluded volumes, and no throat can be computed for path $j$.

[41] As a result of conditions 1 and 2, the cross-section list for path $p$ may have fewer than $n_{p}$ entries (in fact it may be empty). Once a cross-section list has been completed for a path, it is sorted, smallest to largest, by magnitude of $A_{v}$. For the path, the surface with the smallest area is considered the current throat candidate and its area and barrier are referred to as the current throat area and barrier. However, the remaining computed throats in the cross-section list are retained as possible contenders for the path throat.

[42] The throat-finding algorithm consists of three steps followed by sequential iteration of a modified first and third step.

[43] Step 1. The cross-section list is computed independently for each medial axis path. Independence means that the possible existence of throats on other paths does not affect the computation of the cross-section list for any single path $p$. (Consequently, condition 2 is not applied.) At the completion of step 1, paths fall into two groups: (G1a) those that have a current area and (G1b) those that do not (cross-section list empty due to application of condition 1). The paths in G1a are ordered according to the value, smallest to largest, of their current areas.

[44] Step 2. A subset of paths whose throats do not intersect each other are declared as follows. The current throat, area, and barrier of the first path in G1a (the sorted path with the smallest current area) are declared to be the throat, area, and barrier for path 1 . The algorithm loops over the remainder of the sorted path list in G1a. For each subsequent path, $i$, the throat barriers corresponding to the lowest 5th percentile in area are examined. The smallest area barrier intersecting none of the previously declared throat barriers, together with its throat and throat area, are declared to characterize the throat for path $i$. Otherwise, no throat is declared for path $i$ at this point. Condition 2 is applied during this step; if the declared throat barrier for path $k$ passes through a medial axis path $k+j$, then its exclusion volume is applied to the cross-section list for path $k+j$, which is modified accordingly. At the end of step 2, paths now fall into three groups: (G2a) those that have a declared (nonintersecting) throat, (G2b) those that have current area (throat as yet undeclared), and (G2c) those that have no cross-section list. G2b comprises those paths in G1a whose throat barriers intersect with those of other paths. G2c comprises those paths in G1b plus paths whose cross-section lists were eliminated in step 2 as a result of application of condition 2 .

[45] Step 3. In step 3, the algorithm declares throats which are terminated by other throats. It examines only the paths in group $\mathrm{G} 2 \mathrm{~b}$, addressing them in the same order as in step 2. Let path $j$ be one such path. The planar dilation algorithm is rerun for the voxels comprising the lowest 5th percentile of entries in the cross-section list for path $j$. However, now the planar dilation is blocked whenever barrier voxels from previously declared throats (those in group G2a plus those throats that become declared as the paths in group $\mathrm{G} 2 \mathrm{~b}$ are processed) are encountered. Condition 2 is applied using all declared throats in step 3 . From the throats reconstructed out of this 5 th percentile sample, that with the smallest area (together with its barrier) is declared to comprise the throat for path $j$. At the end of step 3, paths now fall into three groups: (G3a) those that have a declared (nonintersecting) throat, (G3b) those that have a declared intersecting throat, and (G3c) those that have no crosssection list. G3c comprises those paths in G2c plus paths whose cross-section lists were eliminated in step 3 as a result of application of conditions 1 and 2.

[46] Step 1'. The planar dilation algorithm is rerun on all paths in group G3c. For purposes of this dilation, all paths in this group are treated as independent of each other; however their dilated throat candidates are terminated by declared throats in groups G3a and G3b. Condition 2 for all declared throats is applied during the planar dilation of paths in G3c. At the end of step $1^{\prime}$, paths in group G3c fall in two subgroups: $\left(\mathrm{G1}^{\prime} \mathrm{a}\right)$ those with cross-section lists with current areas and $\left(\mathrm{G}^{\prime} \mathrm{b}\right)$ those having no current area (the result of application of conditions 1 and 2). The paths in G1'a are sorted according to current area.

[47] Step 3'. Step 3 is rerun on the sorted paths in G1'a.

[48] Steps $1^{\prime}$ and $3^{\prime}$ are iterated until group $\mathrm{G} 1^{\prime} \mathrm{a}$ is empty. 
[49] Steps 1-3' locate, where possible, the smallest area (primary) throat on each path. We allow for the possibility that a path might have more than one throat as in Figure 2a. Multiple throats are located on any path only after primary throats have been found on all paths. Let $p$ be a path for which the primary throat is $v_{1}, \theta_{v_{1}}, \varphi_{v_{1}}, A_{v_{1}}, B_{v_{1}}$. Consider the remainder of the cross-section list for $p$. Apply the exclusion volume around the throat voxel $v_{1}$, as well as enforce any exclusion volumes from other throats (condition 2) that may intersect $p$. The barriers and cross-section areas for the (nonexcluded) remainder of the cross-section list must also be recomputed as throats that have been declared in earlier steps may cut through these cross sections. Moving along $p$ on one side of $v_{1}$, locate the first (two-sided limit) minimum cross-section area located outside of any exclusion region. This is then declared to be the next throat $v_{2}, \theta_{v_{2}}, \varphi_{v_{2}}, A_{v_{2}}, B_{v_{2}}$ on $p$. Establish the exclusion volume around $v_{2}$. This procedure is iterated along $p$, in both directions from $v_{1}$, until all local minima (two-sided limit) in nonexcluded regions have been located.

[50] Once all possible throats have been declared, a pore-throat network is constructed whereby the entire void volume is distributed into individual pores separated by planar throat surfaces having no volume. During the network construction, postprocessing modification of intersecting throats occurs. In line with the discussion of Figure 8 , each throat surface must separate exactly two pores. Therefore, if one throat serves as a boundary for another, the blocking throat is split appropriately.

[51] This procedure is illustrated in Figure 11. As indicated in Figure 11a, a barrier voxel such as $v_{2,3}$ may border more than two pores. Such barrier voxels are flagged as throat intersection voxels. A throat barrier is split along its set of such intersection voxels (Figure 11b). As a voxel is the unit of digitization, we do not subdivide an intersection voxel. Rather, we assign an intersection voxel to each of the barriers of the intersecting throats (i.e., $v_{2,3}$ is assigned to each of barriers 1, 2, and 3 in Figure 11b), but assign an appropriate partial area to each of the throats intersecting in the barrier voxel. Figure 12 illustrates this procedure with an example. Initially (Figure 12a), throat $T_{1}$ blocks $T_{2}$. Throat $T_{1}$ is split into $T_{1}$ and $T_{3}$ (Figure $12 \mathrm{~b}$ ); $T_{1}$ and $T_{3}$ each are assigned one-half of area $A_{1}$. Throat $T_{2}$ is assigned half of area $A_{2}$. (These area assignments are reasonable

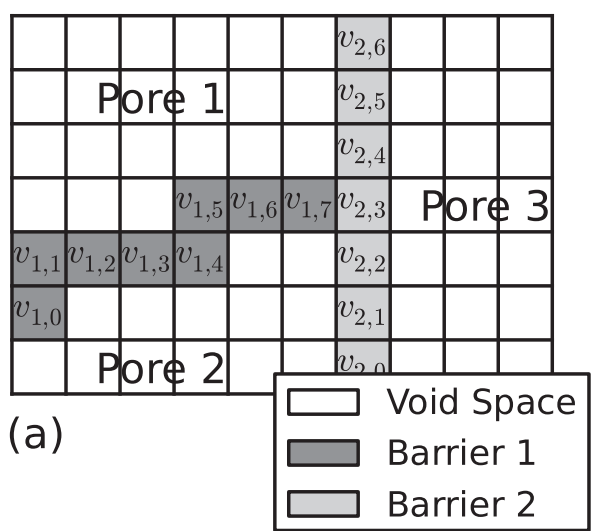

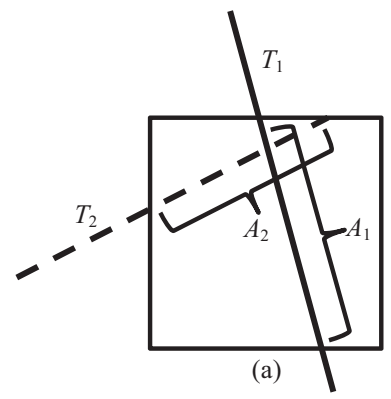

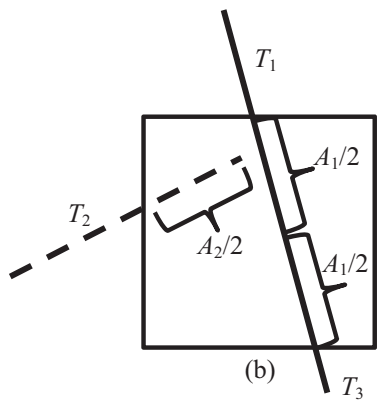

Figure 12. 2-D illustration of throat splitting and the assignment of approximate throat areas in an intersection barrier voxel. (a) $A_{1}$ and $A_{2}$ are the areas of intersection of the barrier voxel with the two throats $T_{1}$ and $T_{2}$. (b) $T_{1}$ is split into throats $T_{1}$ and $T_{3}$; half of area $A_{1}$ is assigned to each of these throats. Half of area $A_{2}$ is assigned to throat $T_{2}$.

approximations that avoid a detailed geometrically accurate computation.)

[52] The existence of throat barrier intersection voxels, which now form part of the boundary of more than one throat, must be taken into account when computing face polygons and throat perimeters. Consistent with the above approximation used for throat area assignments in intersection voxels, face polygons are assigned to run through the centers of barrier intersection voxels as illustrated in Figure 10b (solid line). To reduce staircasing affects, we then modify the face polygons by connecting midpoints-producing the throat perimeter (dashed line).

[53] We have observed that, in the resulting pore-throat network, a pore may have a throat with an area that is large relative to the total surface area of one of the two pores it separates. Based on models of hemispherical pores, we remove a throat if its area exceeds $1 / 3$ of the surface area of either of the two pores it separates. This, of course, results in the merger of the two pores.

\section{Numerical Computation}

[54] The throat-finding algorithm was applied to the pore network in Figure 5. The assumption of planar throats is

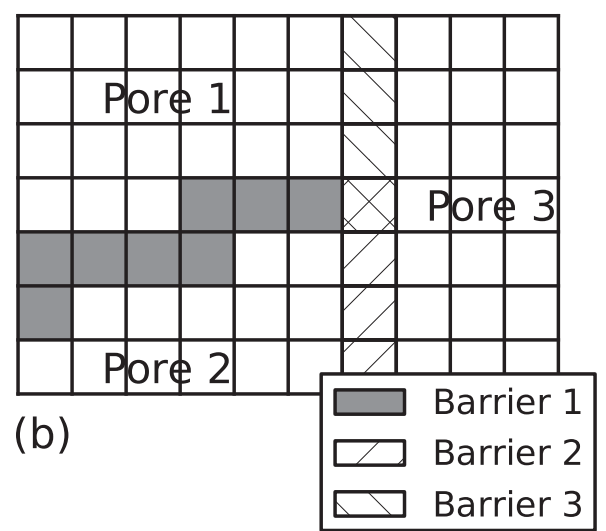

Figure 11. Illustration of the postprocessing step that splits throat barriers. In (a), barrier 2 was constructed first, blocking barrier 1. In (b), the configuration has been split into three barriers. 


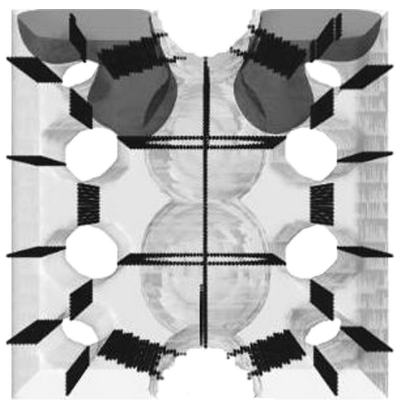

(a)

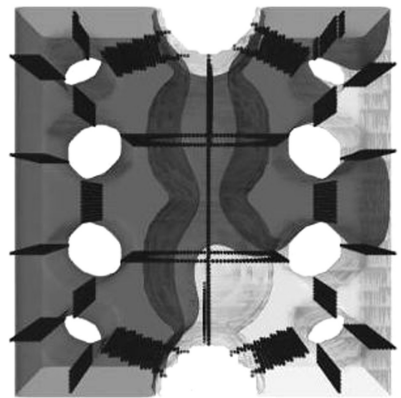

(d)

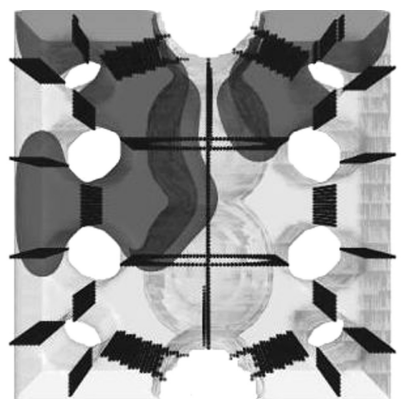

(b)

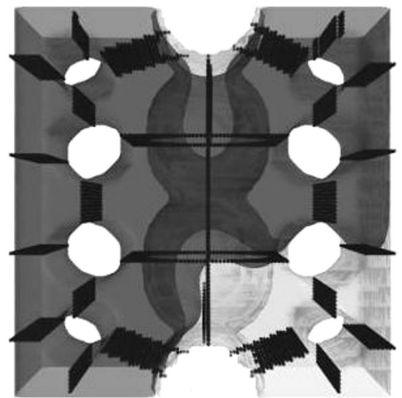

(e)

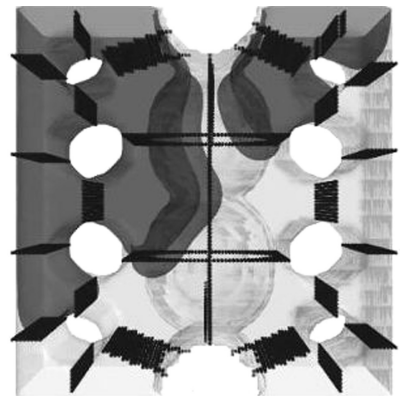

(c)

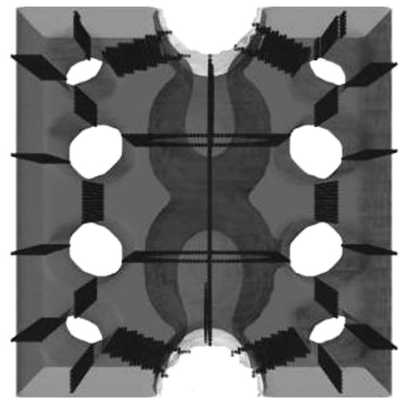

(f)

Figure 13. LSMPQS simulation of drainage flow (top to bottom) through the network of Figure 5. The nonwetting fluid, $f_{n}$ is dark gray, $f_{w}$ is light gray. The computed planar throat surfaces are black. There are 32 throats, nine on the left side, nine on the right, and 14 running through the middle.

appropriate for the geometry of this network; the computed throats - there are 32 of them-are shown in Figure 13a. Also shown in Figure 13 are snapshots of an LSMPQS drainage simulation (flow from top to bottom). The drainage simulation clearly demonstrates how intersecting throats "act" as capillary barriers.

[55] In Lindquist et al. [2000], pore volume and throat area distributions were computed using the algorithm PLS (which allows for curved throat surfaces) for a suite of four XCMT images of Fontainebleau sandstone samples. The analyzed samples were $550 \times 550 \times 511$ voxels, with voxel size $5.7 \mu \mathrm{m}$. Sample porosities were $7.5 \%, 13 \%$, $18 \%$, and $22 \%$. We recomputed the pore-throat network using the new planar dilation (PD) algorithm. Each algorithm comprises two major phases, identification of all throats followed by construction of a pore-throat network. Some modification of the identified throats occurs during the network construction phase. For the PD, this consists of splitting of "blocking" throats and elimination of large area throats. For the PLS algorithm, this consists of deleting any throat that separates more than two pores. We therefore compare the results of the PLS and PD throatfinding algorithms at the end of each of these two phases.

[56] Figure 14 summarizes the throat number density (number of throats per $\mathrm{mm}^{3}$ of sample) identified in the four Fontainebleau samples by the two algorithms at the end of each of the two phases. The throat density increases with the porosity of the sandstone. After the throat identification phase, the PLS algorithm locates 3-5\% more throats than the PD algorithm. However in its pore-network construction phase, the PLS algorithm rejects roughly $10 \%$ of the throats, whereas the pore-throat network construction phase of the PD algorithm sees a net gain of throats through throat splitting. Consequently, after the network construction phase, the PD algorithm has a throat number density that exceeds that of the PLS algorithm by $13-22 \%$.

[57] Figure 15 compares the throat area distributions for the lowest $(7.5 \%)$ and highest $(22 \%)$ porosity Fontainebleau samples after the throat identification phase (left plots) and after the network construction phase (center

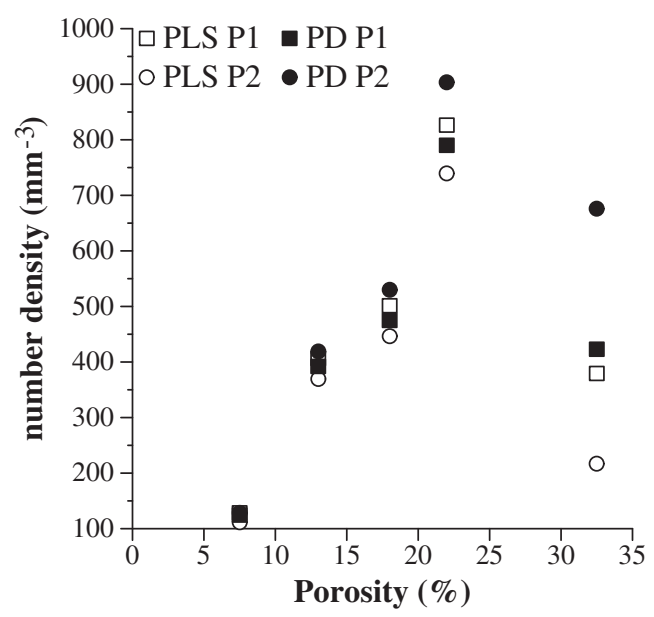

Figure 14. Throat number density as a function of sample porosity as determined by the PLS and PD algorithms after the throat identification phase (P1) and after the pore-throat network construction phase $(\mathrm{P} 2)$. 


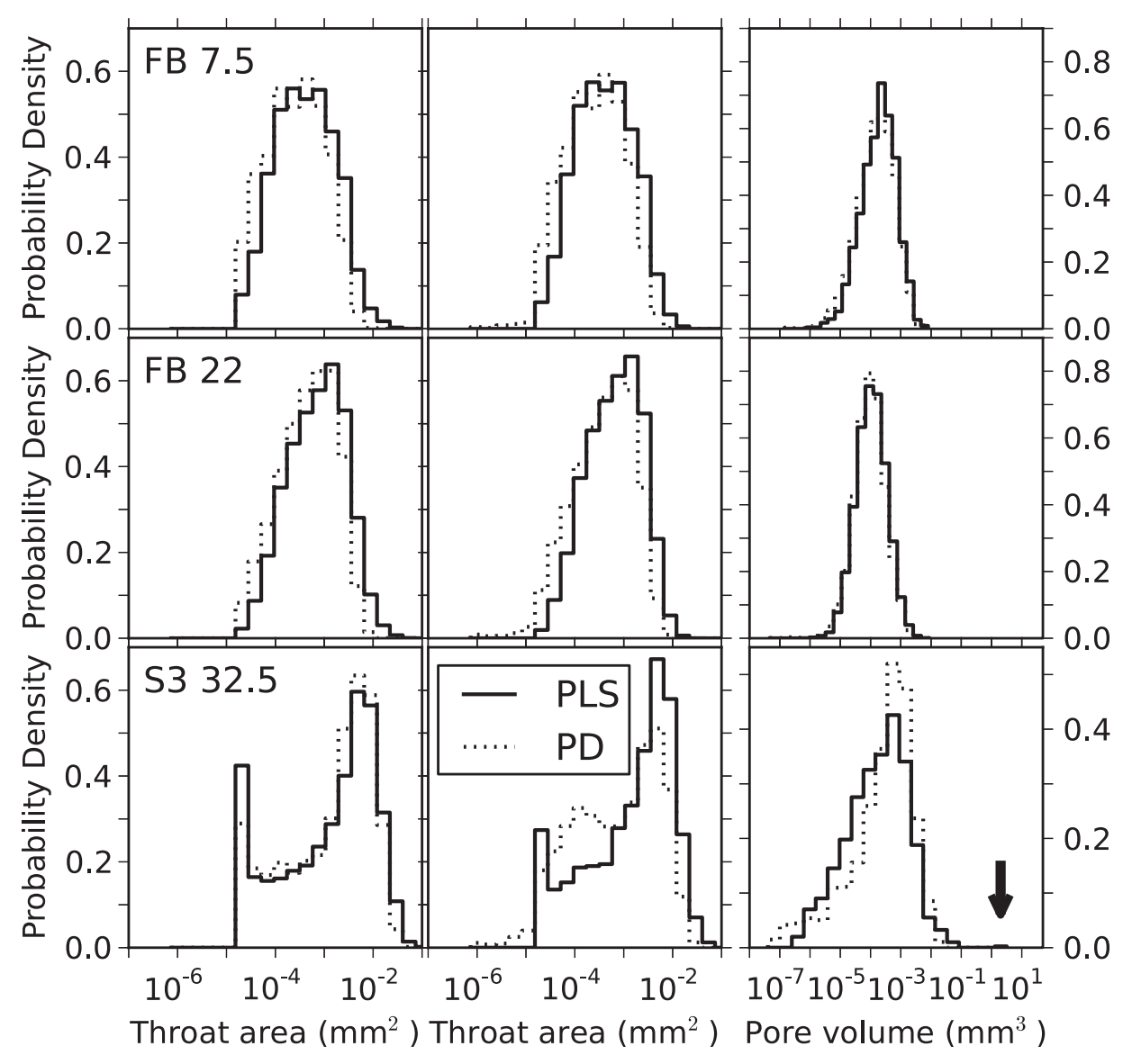

Figure 15. Throat area and pore volume distributions for the $7.5 \%$ and $22 \%$ porosity Fontainebleau sandstone images (first and second rows) and a 32.5\% porosity sample S3 image (third row) using the new PD algorithm compared to the results of the PLS algorithm. The graphs on the left present the distribution of throat area identified during the throat identification phase of each algorithm; the graphs in the center present the distribution of throat areas after the pore-throat network construction phase.

plots). While the overall shape of the distributions is similar, the PLS distribution is shifted slightly to a larger area. At the end of the network construction phase, there is a slight difference in the distribution of smaller throat sizes due to the splitting of blocking (intersecting) throats in the PD algorithm. Such throats are discarded in the network construction phase of the PLS algorithm.

[58] The pore volume distributions, available after the pore-network construction phase, are compared in the right plots of Figure 15. The distributions are very similar between the two algorithms.

[59] The performance of the PLS and PD algorithms was also compared on an unconsolidated sample (S3), 32.5\% porosity, of Accusand grains screened to have diameter between 200 and $300 \mu \mathrm{m}$. The quartz grains were packed in a column of height $8.8 \mathrm{~cm}$ and inner diameter $3.1 \mathrm{~mm}$ using a wet packing technique and a continuous grain stream while vibrating the column to remove gas bubbles and achieve uniform grain packing. The ends of the column were covered by $5 \mu \mathrm{m}$ Spectra/Mesh to retain particles. After packing, the column was subjected to flow of uncontaminated groundwater for two full days at room temperature to stabilize the grain packing and remove any dispersible particles. To minimize depositional effects due to gravity, during this saturation period a constant upward flow rate of $0.67 \mathrm{ml} /$ day (approximately $1 \mathrm{PV} /$ day) was maintained using a peristaltic pump. A section of the sample was imaged at $3.96 \mu \mathrm{m}$ voxel size using XCMT. The analyzed image size was $600 \times 600 \times 700$ voxels.

[60] Also shown, respectively, in Figures 14 and 15 are the throat number density, throat area, and pore volume distribution comparisons obtained for sample S3. In spite of the increased porosity, the throat number density of this unconsolidated sample is comparable to Fontainebleau samples of smaller porosity. After the throat identification phase, the throat number density is $11 \%$ smaller for the PLS algorithm. After the pore-network construction phase, the handling of intersecting throats (retained and subdivided in the PD algorithm, rejected in the PLS algorithm) results in marked differences in the throat number densities. The throat area distributions after throat identification are similar for the two algorithms; after the pore-throat network phase, there are marked differences between the throat area distributions. Rejection of the possibility of intersecting throats by the PLS algorithm has drastic consequences in pore partitioning in S3. The pore volume distributions for S3 show more distinct differences than the lower porosity Fontainebleau comparisons. The porenetwork construction for S3 from the PLS algorithm resulted in a largest pore having volume $2.03 \mathrm{~mm}^{3}$. In 


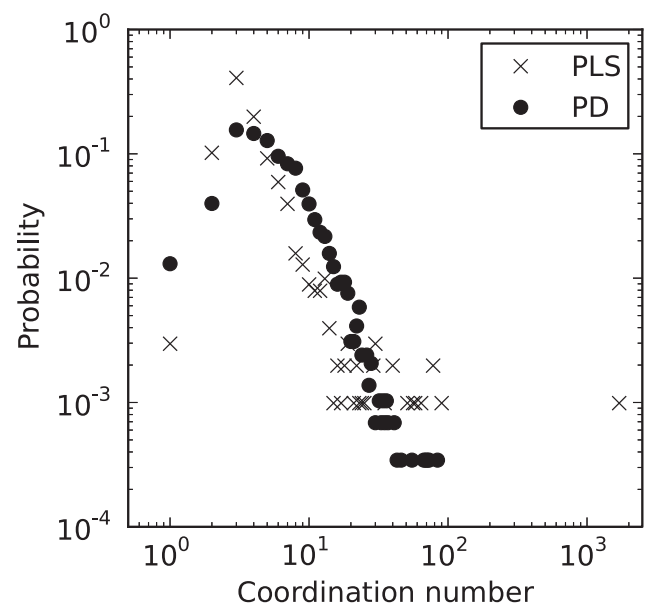

Figure 16. Comparison of pore coordination number distribution computed from the XCMT image of sample S3 using algorithms PD and PLS.

contrast, the largest pore constructed by the PD algorithm had a volume of $0.03 \mathrm{~mm}^{3}$.

[61] The production of large pores by the PLS algorithm in high-porosity samples is the most significant consequence of neglecting intersecting throats. As shown in Figure 16, such pores have very large coordination number. The PLS algorithm also produces significantly more pores having coordination number 2 and 3 . The increase of the number of coordination number 1 pores in the PD algorithm is the result of a subtle effect. Throat areas are computed from the planar throat surfaces which cut through barrier voxels; individual pores are identified using the throat barriers - which are digitized at the voxel level. Thus, the plane surface passing through a barrier voxel may not come into contact with the grain surface while the barrier voxel itself may contact the grain surface. Since barrier voxels determine distinct pores, this subtle effect may cause a pore to be split into two pieces, with the "split-off" piece commonly being a small, coordinationnumber 1 pore.

[62] Figure 17 plots the percentage of all throats that intersect as a function of porosity of the investigated sample. Over the porosity range $7.5-22 \%$, the percentage of intersecting throats increased at the rate of $0.3 \%$ per percent porosity change. Over the porosity range $22-32.5 \%$ intersecting throats increased at the significantly greater rate of $1.8 \%$ per percent porosity change, indicating the need for throat-finding algorithms that capture intersecting throats. For sample S3, 27.95\% of all throats intersected with another.

\section{Discussion}

[63] One consequence of (the standard) definition, D1, for a throat is that entry menisci during drainage occur in the downstream vicinity of a throat; the deviation from throat location depending on wetting angle. In addition, depending on flow direction, no entry meniscus will occur at some throat locations. We have shown that throats so defined may intersect each other and propose a criterion (T5) for differentiating throats that do intersect. We have

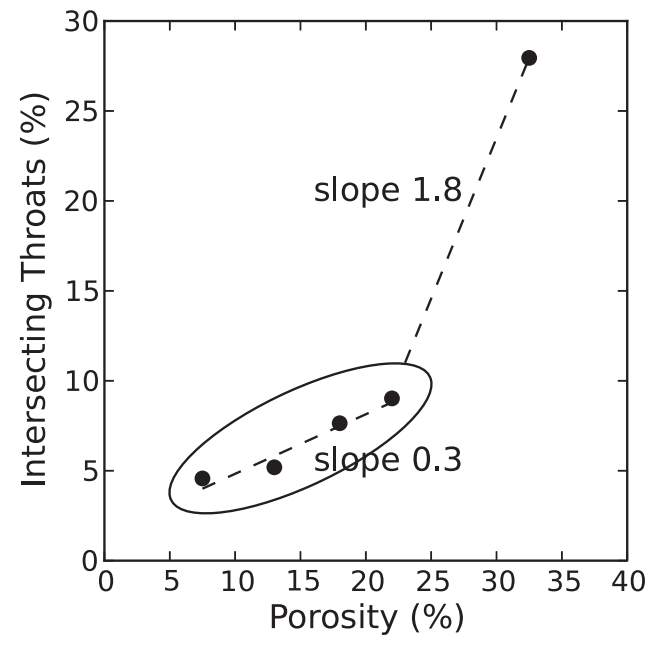

Figure 17. Observed increase in the frequency of intersecting throats (expressed as a percentage of all throats) with porosity.

shown by numerical computation (section 2) that these intersecting throats do indeed correspond to locations of capillary barriers to flow in intersecting directions.

[64] A pore-network model is a standard construct used to visualize, simplify, and characterize the void space network in a porous medium, especially for the extremely random networks found in geologic media. The recognition that throat surfaces in such a network intersect each other, significantly so for porosities exceeding $20 \%$, has implications for this construct. As throat surfaces approximate capillary barriers in drainage processes, one implication is that drainage flow potentially bifurcates where throat surfaces intersect. This has consequences (e.g., temporal order of wetting film formation) for network flow model computations.

[65] The throat-finding algorithm proposed here explicitly captures intersecting throats. In the interests of speed, throats are approximated as planar surfaces; it would be useful to have a fast algorithm to better approximate curved minimal-area surfaces. Our studies with this algorithm indicate that the percentage of intersecting throats increases dramatically above $20 \%$ porosity. However, our highporosity sample is an unconsolidated sand pack, while our lower porosity samples are consolidated sandstones. We do not know how strongly the rate of throat intersection depends on consolidation of the grains.

[66] Acknowledgments. We thank Masa Prodonovic (U.T. Austin) for access to, and assistance with, the software package LSMPQS. This work is supported by the Research Foundation of SUNY (DK) and the US Department of Energy under contracts DE-FG02-92ER14261 and DEFG02-09ER64747.

\section{References}

Bear, J. (1972), Dynamics of Fluids in Porous Media, 764 pp., Dover, New York.

Blunt, M. J. (2001), Flow in porous media-pore-network models and multiphase flow, Curr. Opin. Colloid Interface Sci., 6, 197-207.

Dullien, F. A. L. (1992), Porous Media: Fluid Transport and Pore Structure, 574 pp., Academic, San Diego, Calif.

Edelsbrunner, H., and J. Harer (2008), Persistent homology-A survey, in Surveys on Discrete and Computational Geometry. Twenty Years Later, edited by J. E. Goodman, J. Pach, and R. Pollack, pp. 257-282, Am. Math. Soc., Providence, R. I. 


\section{KIM ET AL.: A RE-EXAMINATION OF THROATS}

Frette, O. I., and J. O. Helland (2010), A semi-analytical model for computation of capillary entry pressures and fluid configurations in uniformlywet pore spaces from 2D rock images, Adv. Water Resour., 33, 846-866.

Gao, S. Y., J. N. Meegoda, and L. M. Hu (2012), Two methods for pore network of porous media, Int. J. Numer. Anal. Methods Geomech., 36(18), 1954-1970.

Glantz, R., and M. Hilpert (2007), Dual models of pore spaces, Adv. Water Resour., 30(2), 227-248.

Glantz, R., and M. Hilpert (2008), Tight dual models of pore spaces, $A d v$. Water Resour., 31(5), 787-806.

Hwang, S. K. (1977), The Gauss equation in capillarity, Z. Phys. Chem., $105,225-235$.

Joekar-Niasar, V., S. M. Hassanizadeh, L. J. Pyrak-Nolte, and C. Berentsen (2009), Simulating drainage and imbibition experiments in a highporosity micromodel using an unstructured pore network model, Water Resour. Res., 45, W02430, doi:10.1029/2007WR006641.

Koplik, J., and T. J. Lasseter (1985), Two-phase flow in random network models of porous media, SPEJ Soc. Pet. Eng. J., 25(1), 89-100.

Kovscek, A. R., and C. J. Radke (1996), Gas bubble snap-off under pressure-driven flow in constricted noncircular capillaries, Colloids Surf. A, 117(1-2), 55-76.

Lee, T. C., R. L. Kashyap, and C. N. Chu (1994), Building skeleton models via 3-D medial surface axis thinning algorithms, CVGIP: Graphical Models Image Process., 56(6), 462-478.

Lenormand, R., C. Zarcone, and A. Sarr (1983), Mechanisms of the displacement of one fluid by another in a network of capillary ducts, J. Fluid Mech., 135, 337-353.

Lindquist, W. B. (2006), The geometry of primary drainage, J. Colloid Interface Sci., 296(2), 655-668.

Lindquist, W. B., and A. Venkatarangan (1999), Investigating 3D geometry of porous media from high resolution images, Phys. Chem. Earth Part A, 24(7), 593-599.

Lindquist, W. B., A. Venkatarangan, J. Dunsmuir, and T. F. Wong (2000), Pore and throat size distributions measured from synchrotron X-ray tomographic images of Fontainebleau sandstones, J. Geophys. Res., 105(B9), 21,509-21,527.
Mayer, R. P., and R. A. Stowe (1965), Mercury porosimetry-breakthrough pressure for penetration between packed spheres, J. Colloid Interface Sci., 20(8), 893-911.

Morrow, N. R. (1970), Physics and thermodynamics of capillary action in porous media, Ind. Eng. Chem., 62(6), 32-56.

Oh, W., and W. B. Lindquist (1999), Image thresholding by indicator kriging, IEEE Trans. Pattern Anal. Mach. Intell., 21(7), 590-602.

Princen, H. M. (1969a), Capillary phenomena in assemblies of parallel cylinders. I. Capillary rise between 2 cylinders, J. Colloid Interface Sci., 30(1), 69-75.

Princen, H. M. (1969b), Capillary phenomena in assemblies of parallel cylinders. 2. Capillary rise in systems with more than 2 cylinders, J. Colloid Interface Sci., 30(3), 359-371.

Prodanovic, M., and S. L. Bryant (2006), A level set method for determining critical curvatures for drainage and imbibition, J. Colloid Interface Sci., 304(2), 442-458.

Prodanovic, M., W. B. Lindquist, and R. S. Seright (2006), Porous structure and fluid partitioning in polyethylene cores from 3D X-ray microtomographic imaging., J. Colloid Interface Sci., 298(1), 282-297.

Raoof, A., and S. M. Hassanizadeh (2012), A new formulation for porenetwork modeling of two-phase flow, Water Resour. Res., 48, W01514, doi:10.1029/2010WR010180.

Shaeri, M. R., S. Beyhaghi, and K. M. Pillai (2013), On applying an external-flow driven mass transfer boundary condition to simulate drying from a pore-network model, Int. J. Heat Mass Transfer, 57(1), $331-344$.

Shin, H., W. B. Lindquist, D. L. Sahagian, and S. R. Song (2005), Analysis of the vesicular structure of basalts, Comput. Geosci., 31(4), 473-487.

Sholokova, Y., D. Kim, and W. B. Lindquist (2009), Network flow modeling via lattice-Boltzmann based channel conductance, $A d v$. Water Resour., 32(2), 205-212.

Silin, D., and T. Patzek (2006), Pore space morphology analysis using maximal inscribed spheres, Physica A, 371(2), 336-360.

van der Marck, S. C., T. Matsuura, and J. Glas (1997), Viscous and capillary pressures during drainage: Network simulations and experiments, Phys. Rev. E, 56(5), 5675-5687. 